\title{
Three-dimensional microstructuring of yttrium aluminum garnet crystals for laser active optofluidic applications
}

\author{
D. Choudhury, ${ }^{1,2}$ A. Rodenas, ${ }^{1, a)}$ L. Paterson, ${ }^{2}$ F. Díaz, ${ }^{3}$ D. Jaque,${ }^{4}$ and A. K. Kar ${ }^{1}$ \\ ${ }^{1}$ Institute of Photonics and Quantum Sciences, Heriot-Watt University, Edinburgh EH14 4AS, Scotland, \\ United Kingdom \\ ${ }^{2}$ Institute of Biological Chemistry, Biophysics and Bioengineering, Heriot Watt University, \\ Edinburgh EH14 4AS, Scotland, United Kingdom \\ ${ }^{3}$ Física i Cristal-lografia de Materials i Nanomaterials(FiCMA-FiCNA). Universitat Rovira i Virgili (URV), \\ Campus Sescelades cl Marcel-lí Domingo, s/n. E-43007 Tarragona, Spain \\ ${ }^{4}$ Fluorescence Imaging Group, Facultad de Ciencias, Universidad Autónoma de Madrid, Cantoblanco, \\ Madrid, Spain
}

(Received 6 June 2013; accepted 5 July 2013; published online 22 July 2013)

\begin{abstract}
We demonstrate three-dimensional microstructuring in a neodymium doped yttrium aluminum garnet (Nd:YAG) crystal. Spatially well-defined hollow microstructures deeply embedded within the material are shown to result from the creation of a pre-damage state within the Nd:YAG crystal network that is validated using luminescence and Raman analyses of the structures. This opens up the potential of fabricating next-generation optofluidic devices in optical gain materials. (C) 2013 AIP Publishing LLC. [http://dx.doi.org/10.1063/1.4816338]
\end{abstract}

The future growth in the field of optofluidics depends upon exploring materials with unique properties as relevant alternatives to existing substrate materials. Such alternative materials for biological and chemical optofluidic applications must be prudently selected based on important properties such as chemical stability, biocompatibility, mechanical strength, well-characterised optical properties, and availability before being investigated for optimised microfabrication. In addition, the material must also be suitable for the development of optofluidic architectures to controllably deliver and collect light within a micro-scale environment. In current optofluidic applications light is typically delivered into the device either using free-space optics or fiber-coupling methods. Applications that require different wavelengths need additional light sources that in turn impose increased space and cost requirements. In this regard, substrate materials that enable light sources to be integrated within the device are essential to the progress of optofluidics. Efforts to generate light on-chip have so far been channeled towards the incorporation of fluorescent species, such as Rhodamine $6 \mathrm{G}$ or its variants, in organic solutions that are confined within microfluidic channels suitably designed in various resonator configurations. ${ }^{1}$ Dye based methods are well suited for the generation of visible wavelengths typically ranging between $\approx 560$ and $600 \mathrm{~nm}^{2,3}$ Recently, ultraviolet whispering gallery mode lasing at a single frequency has been demonstrated at $386.75 \mathrm{~nm}$ using a commercial near-UV laser dye in dimethyl sulfoxide. ${ }^{4}$ The on-chip generation of dual wavelengths of light has also been demonstrated using microfluidic dye lasers in collinear ${ }^{2}$ as well as parallel geometries. ${ }^{5}$ However, the wavelength tunability of microfluidic dye lasers is restricted by the emission wavelengths of the fluorescent molecules. Moreover, the toxicity aspects of the dyes and solvents cannot

\footnotetext{
a) Present address: Física i Cristal-lografia de Materials i Nanomaterials (FiCMA-FiCNA). Universitat Rovira i Virgili (URV), Campus Sescelades c/ Marcel-lí Domingo, s/n. E-43007 Tarragona, Spain
}

be trivialized especially with regard to biological analysis. The application of such light sources is yet to be achieved in the near-infrared (NIR) region of the electromagnetic spectrum, which constitutes an important spectral window for biological analytics. We address the aforementioned requirement by introducing the solid-state optical gain media Nd:YAG as an alternative class of materials for optofluidic applications, where microstructured channels can potentially be integrated with optically active waveguides.

The nonlinear interactions of femtosecond ( $f s$ ) laser pulses with transparent dielectric materials have been extensively studied in the last few decades with an aim to apply the knowledge to high precision microfabrication ( $\mu$-fabrication) applications. The phenomenon of selective chemical etching in fs pre-irradiated micro-volumes was first demonstrated in photosensitive glass ${ }^{6}$ and then in fused silica glass. ${ }^{7}$ Since then, it has been developed into a well established and controllable method to fabricate almost arbitrary three-dimensional (3D) structures inside these glasses, such as $\mu$-fluidic devices, ${ }^{8-13}$ or $\mu$-mechanical sensors. ${ }^{14}$ One limitation of the technique is, however, the fact that so far it only works in glasses. In the case of crystalline dielectrics, it has been found to be much more difficult to apply the technique with the same level of results. Although there are various experimental reports of wet etching laser processed $\mu \mathrm{m}$-size features in crystals such as crystalline quartz and sapphire, ${ }^{15,16}$ and of even sub- $\mu \mathrm{m}$ features in lithium niobate, ${ }^{17}$ the possibility of fabricating $\mu$-fluidic devices with functional 3D $\mu$-structures inside optical materials such as solid state laser crystals is still to be demonstrated. The fabrication of complex structures in laser materials, for example, combining hollow $\mu$-channels with optically active waveguides, will constitute an advanced generation of self-activated optofluidic devices in which light sources are integrated in the device.

In this work, we have demonstrated how $\mu$-fluidic channels are fabricated in Nd:YAG crystals (a paradigm in laser gain media) by the selective wet etching of fs laser directly 


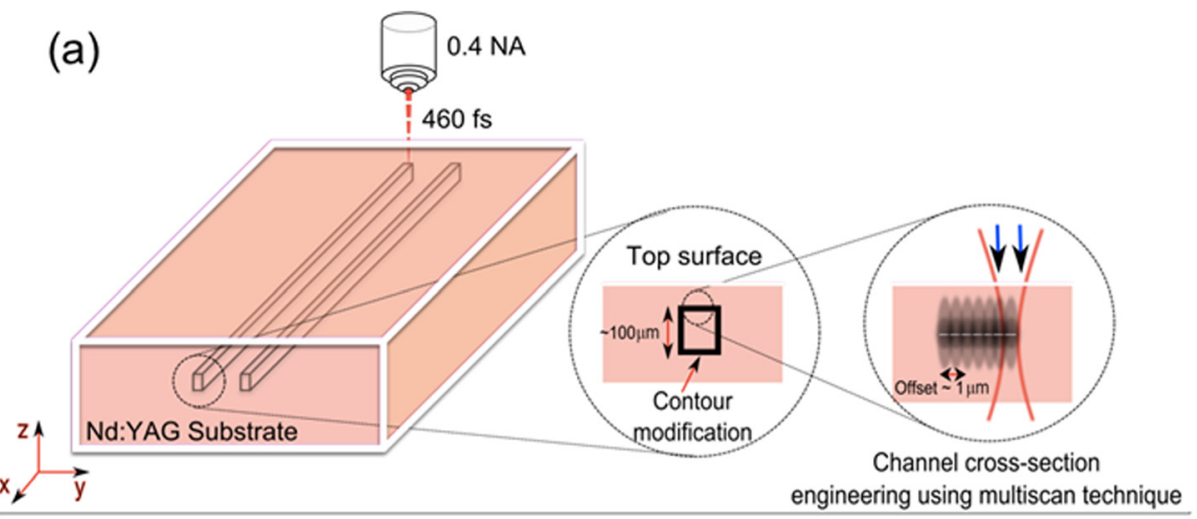

STAGE 1 (b)

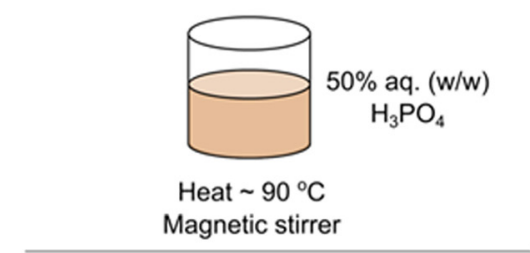

STAGE 2

FIG. 1. Schematic showing the fabrication process (a) The direct laser writing step showing 3D rectangular cross-section microstructuring of the YAG substrate. The writing process involves focusing of $460 \mathrm{fs}$ pulses using a $0.4 \mathrm{NA}$ objective within the volume of the material. Inset shows the contour modification approach using the multiscan technique at an offset of $1 \mu \mathrm{m}$. (b) The selective etching stage using aq. $\mathrm{H}_{3} \mathrm{PO}_{4}$ at a concentration of $50 \%$ (w/w) at $90{ }^{\circ} \mathrm{C}$.

written 3D $\mu$-structures with increased refractive index. We have explored and determined the experimental conditions of both the writing and wet etching steps leading to the damage-free fabrication of $\mu$-channels. Structural images obtained by both $\mu$-luminescence and $\mu$-Raman studies of the irradiated volumes have been used to elucidate the possible origin of the selective etching. In this work, we demonstrate the possibility to fabricate ultra high aspect-ratio fluidic channels within the volume of a laser crystal, a finding that will be instrumental in developing future biophotonic sensing technologies.

For 3D-direct laser writing (DLW), we used a variable repetition-rate Yb-doped master oscillator power amplifier laser system (IMRA $\mu$ Jewel D400) that was set to deliver $460 \mathrm{fs}$ pulses at a central wavelength of $1047 \mathrm{~nm}$. A commercially obtained Nd:YAG crystal (Altechna) with a flatness of $\leq \lambda / 10$ at $632 \mathrm{~nm}$ and a $\mathrm{Nd}^{3+}$ concentration of 1.1 at. $\%$ was used for this work. The fs pulses were focused within the Nd:YAG crystal using a 0.4 numerical aperture (NA) aspheric lens. The polarization state of the pulses and writing geometry were set at circular and transverse respectively for all experiments. Initial DLW parameter acquisition experiments were performed at a pulse repetition-rate of $100 \mathrm{kHz}$ following our first findings on the DLW of localized 3D refractiveindex increased zones in YAG crystals. ${ }^{18}$ Planar structures were written $100 \mu \mathrm{m}$ below the top surface of the crystal using the multiscan technique, featuring 29 scans with an offset distance of $1 \mu \mathrm{m}$ creating a width of $30 \mu \mathrm{m}$. The translation speeds applied in the $100 \mathrm{kHz}$ experiment ranged between 1 and $8 \mathrm{~mm} \mathrm{~s}^{-1}$ and the pulse energy was maintained below $300 \mathrm{~nJ}$. Severe damage and cracking within the crystal was, however, found as a result of scanning large areas with a high density of modified zones. This fundamental limitation had also been previously observed when highdensity 3D photonic structures at $1 \mathrm{kHz}$ were written in YAG. ${ }^{19}$ By increasing the repetition-rate to $500 \mathrm{kHz}$ we circumvented this limitation. This can be attributed to the higher pulse repetition-rate induced heat accumulation, which relaxes the stress fields generated between modified and unmodified zones. In the $500 \mathrm{kHz}$ regime, the $30 \mu \mathrm{m}$ planar structures revealed lower damage and cracking at pulse energy values $\leq 200 \mathrm{~nJ}$ at translation speeds above $4 \mathrm{~mm} \mathrm{~s}^{-1}$.
A parameter window for the damage free DLW was obtained between pulse energy values of 160 and $170 \mathrm{~nJ}$ and a translation speed of $8 \mathrm{~mm} \mathrm{~s}^{-1}$. These parameters allowed 3D contour designs to be subsequently implemented in the DLW of rectangular cross-section $\mu$-structures deep within the Nd:YAG substrates. Figure 1 shows a scheme of the experimental setup used for the two step process of DLW (Fig. 1(a)) and chemical bath wet etching (Fig. 1(b)). The contour design involves the modification of the perimeter of the microchannels using the multiscan technique using a scan offset of $1 \mu \mathrm{m}$. The length of the microchannels was $1 \mathrm{~mm}$. Prior to immersing the samples in the wet etching bath, the samples were also inspected under optical transmission microscopy. Figure 2(a) shows such an optical transmission cross-sectional view of a directly written $\mu$-structure, before any wet etching. The DLW $\mu$-structures were subsequently wet etched using an aqueous solution of orthophosphoric acid $\left(\mathrm{H}_{3} \mathrm{PO}_{4}\right)$ with a maximum concentration of $50 \%(\mathrm{w} / \mathrm{w})$ at an elevated temperature of $90^{\circ} \mathrm{C}$. The laser modified regions were completely removed after $30 \mathrm{~h}$, leaving behind hollow microstructures inside the Nd:YAG substrates. Figure 2(b) shows a scanning electron microscopy (SEM) image of an etched $\mu$-structure. Excellent optical quality
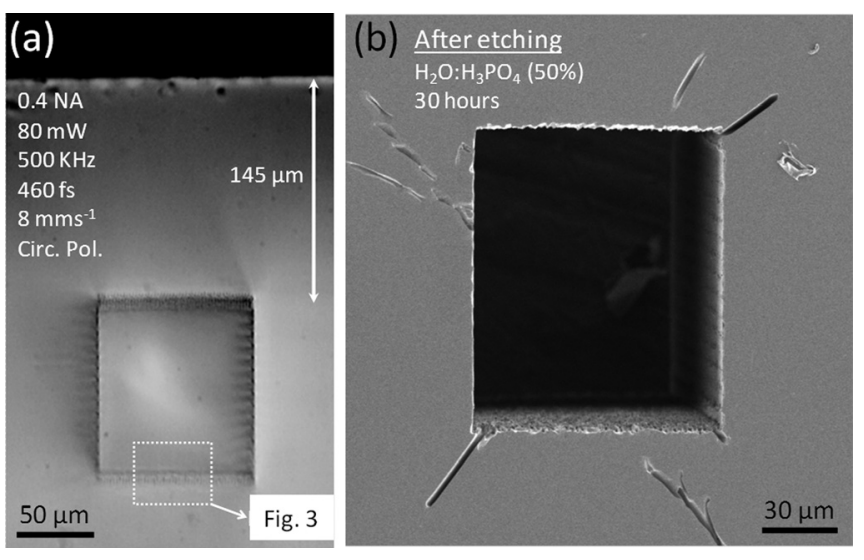

FIG. 2. The 3D contour modified microchannel with a rectangular crosssection. (a) Optical transmission microscope image of the microchannel cross-section. The DLW parameters are shown. (b) SEM image of the microchannel after being etched in $\mathrm{H}_{3} \mathrm{PO}_{4}$ for $30 \mathrm{~h}$ at $90{ }^{\circ} \mathrm{C}$. 
surface roughness was observed in the unmodified crystal even after prolonged etchant exposure.

Confocal $\mu$-fluorescence imaging of the pre-etched structures was performed in order to analyze the origin of the highly selective etching process observed. For this purpose, the $\mathrm{Nd}^{3+}$ luminescence was optically excited using a focused $785 \mathrm{~nm}$ linearly polarized laser beam with a $50 \times(0.75 \mathrm{NA})$ Leica microscope objective mounted on a Renishaw inVia Reflex Leica Raman microscope. The same objective was used to collect the back-scattered luminescence, which, after passing through some filters and apertures, was spectrally analyzed using a high resolution spectrometer. All data were processed using WIRE 3.2 Renishaw software. Figure 3(b) shows a map of the emission integrated area (EIA) of the ${ }^{4} \mathrm{~F}_{3 / 2} \rightarrow{ }^{4} \mathrm{I}_{9 / 2}$ emission line at around $938 \mathrm{~nm}$. It is evident that the EIA is almost unchanged inside the modified regions. This effect is characteristic from this type of index modification, and differs from previous observations on direct laser induced refractive-index decrements as a result of partial lattice amorphization. ${ }^{20}$ The absence of a relevant luminescence reduction at the irradiated volume is a clear indication that severe lattice damage has not been produced. Figure 3(c) shows a slight red-shift of the emission line in the laser modified region in comparison to the bulk crystal, indicating an alteration in the $\mathrm{Nd}^{3+}$ ions crystalline environment. Indeed, according to previous works this red-shift can be associated, in a first order approximation, to the presence of a local lattice compression. Finally, Figure 3(d) shows a marked broadening of the emission peak in the laser modified region, with an increase of 60\% in the FWHM. Following previous results concerning structural imaging of $\mathrm{Nd}$ :YAG crystals and ceramics, it can be concluded that fs irradiation has caused a remarkable increase in the local lattice disorder. Thus, from the fluorescence images of irradiated areas included in Figure 3 it can be concluded that fs irradiation has led the Nd:YAG network to a pre-damaged state characterized by a bond distortion and a certain degree of disorder. The presence of such a pre-damage state has been further confirmed by $\mu$-Raman measurements. For these measurements a $514 \mathrm{~nm}$ linearly polarized laser was used for excitation, and a $100 \times(1.4 \mathrm{NA})$ Nikon objective was used for improved sub-micron spatial resolution. ${ }^{17,19,20}$ Figure 4 shows the spatial variation of the peak frequency (b), peak intensity (c), and width (d) of the $\sim 378 \mathrm{~cm}^{-1}$ characteristic $\mathrm{T}_{2 \mathrm{~g}}(7)$ phonon Raman mode of the YAG network as obtained over the fs irradiated region shown in Figure 4(a). As can be observed, at the irradiated area the Raman mode blue shifts and simultaneously broadens. These two features indicate the presence of a local lattice distortion and partial disorder of the Nd:YAG network. Both features were also concluded from the fluorescence images included in Figure 3. In addition, the Raman peak intensity exhibits a slight decrease, at very localized volumes, which also supports partial damage in the Nd:YAG network attributed to broken and modified bonds. Thus, we conclude from the $\mu$-Raman and $\mu$-luminescence studies that in our irradiation conditions, fs pulses drive the Nd:YAG network to a locally modified crystalline state consisting of a distorted Nd:YAG network that is characterized by different inter-atomic distances as well as by the presence of a larger density of lattice defects including broken bonds that could produce micro/nano cracks. We state that when these modified volumes are exposed to an orthophosphoric acid bath at elevated temperatures, the existence of localized micro/nano cracks favor the penetration depth of the acid into the YAG crystal when compared to unmodified volumes. In addition, the presence of distorted bonds could also result in higher chemical reactivity, as already reported in previous works. ${ }^{21,22}$ Thus it can be concluded that the selective etching observed in laser-modified volumes is very likely caused by a combination of these two effects. Nevertheless, further experiments are necessary to achieve a complete characterization of the mechanisms governing selective etching in YAG crystals.
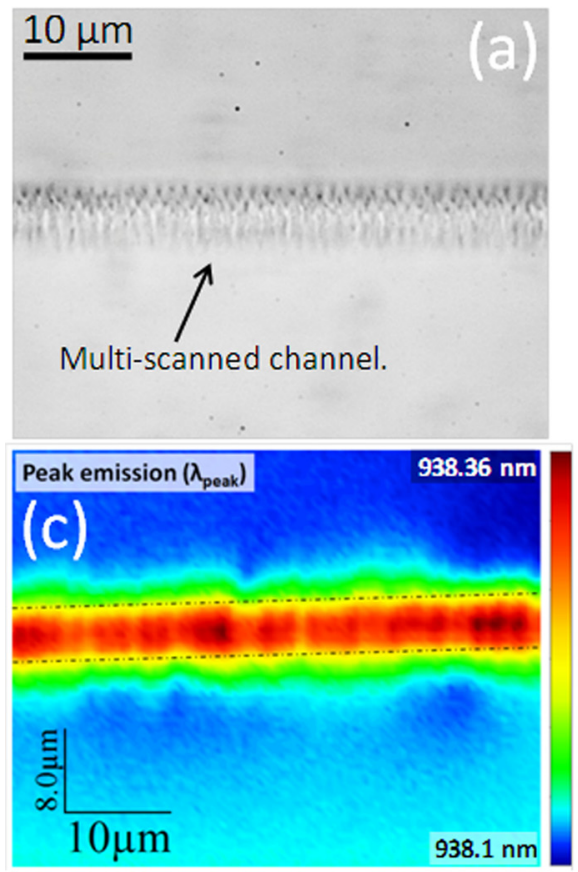
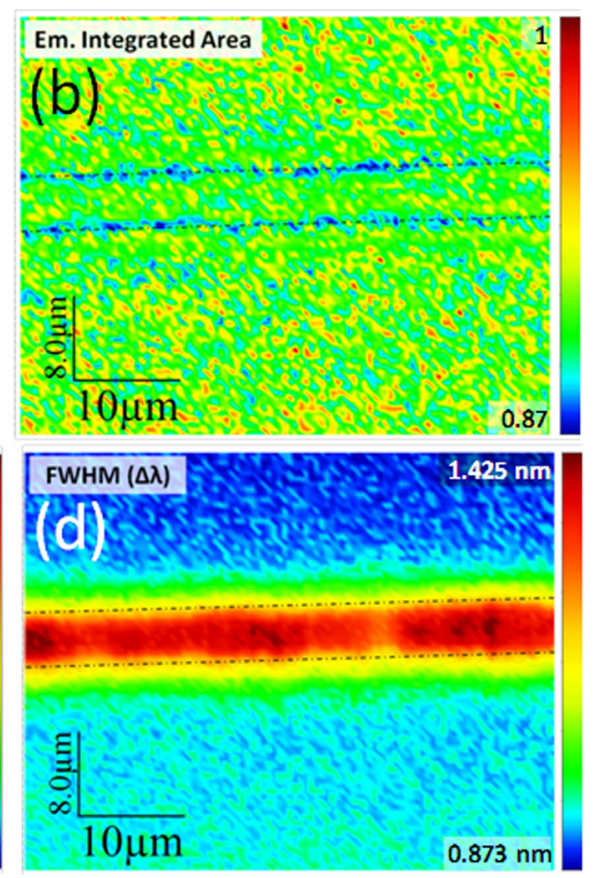

FIG. 3. Confocal $\mu$-luminescence imaging of the fs laser written structure in Nd:YAG. (a) The selected region for performing $\mu$-luminescence analysis as indicated in Figure 2(a). (b) Emission Integrated Area map, (c) peak emission map, (d) peak linewidth map. 

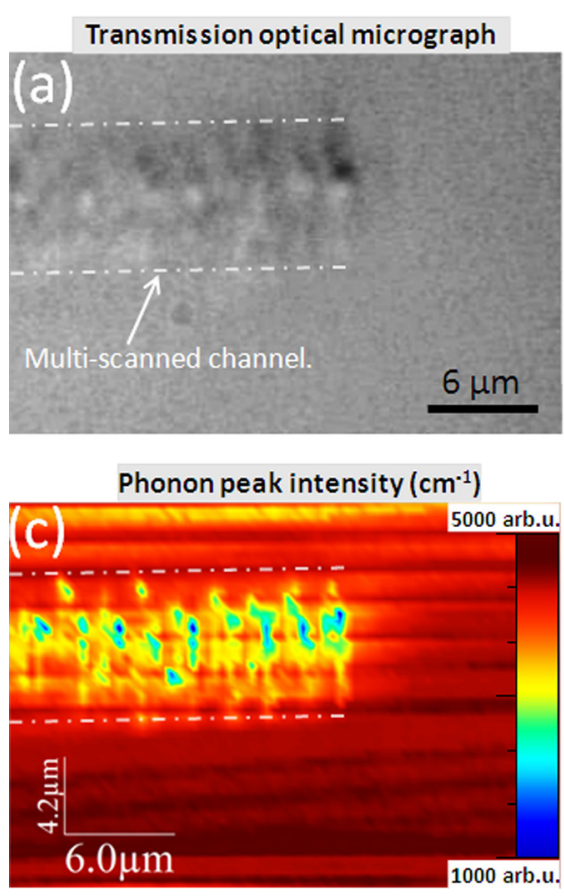
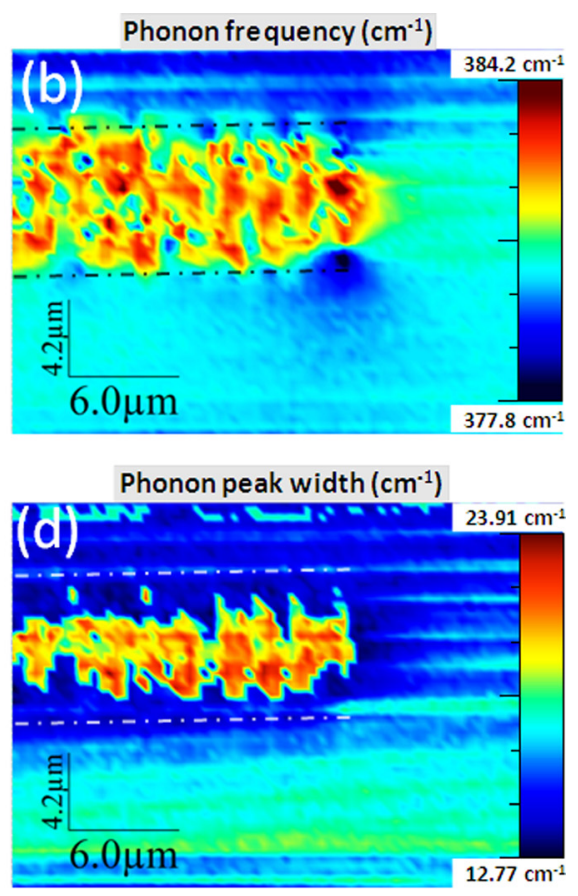

FIG. 4. Confocal $\mu$-Raman imaging of the fs laser written structure in Nd:YAG. (a) The selected region for performing the $\mu$-Raman analysis. (b) Phonon frequency map and (c) phonon peak intensity map, and (d) phonon peak linewidth map.
In summary, we report on the selective material removal with $\mu \mathrm{m}$ size resolution in Nd:YAG crystals using a combination of fs laser writing and wet etching. Fluorescence and Raman imaging of the fs laser irradiated areas have led to the conclusion that, for the pulse energies and repetition rates used in this work, fs pulses have driven the Nd:YAG network to a pre-damaged state that shows a larger selective etching than the unperturbed Nd:YAG network. We have provided in this work the first demonstration of the fabrication of high quality micro-channels in a laser crystal. This proof of concept opens the door to the future development of optofluidic devices capable of internal optical gain.

D. Choudhury acknowledges the support from the Heriot-Watt University Life Sciences Interface Theme Scholarship. A. Rodenas acknowledges financial support from the Spanish Ministerio de Educación under the Programa de Movilidad de Recursos Humanos del Plan Nacional de I + D $+\mathrm{i} 2008 / 2011$ for abroad postdoctoral researchers. D. Jaque thanks Fundación caja Madrid for financial support. This work was also supported by the Universidad Autónoma de Madrid and Comunidad Autónoma de Madrid (Project S2009/ MAT- 1756) and by the Spanish Ministerio de Educacion y Ciencia (MAT2010-16161). The authors wish to acknowledge UK EPSRC (grant number EP/G030227/1) for the IMRA femtosecond MOPA laser system. We thank Renishaw plc. for the gift of the inVia Reflex Leica Raman microscope.

${ }^{1}$ C. Monat, P. Domachuk, and B. J. Eggleton, Nat. Photonics 1, 106-114 (2007).

${ }^{2}$ Q. Kou, I. Yesilyurt, and Y. Chen, Appl. Phys. Lett. 88(9), 091101 (2006).
${ }^{3}$ M. G.-Hansen, S. Balslev, N. A. Mortensen, and A. Kristensen, Microelectron. Eng. 78-79, 185-189 (2005).

${ }^{4}$ X. Tu, X. Wu, M. Li, L. Liu, and L. Xu, Opt. Express 20(18), 19996-20001 (2012).

${ }^{5}$ Y. Cheng, K. Sugioka, and K. Midorikawa, Opt. Lett. 29(17), 2007-2009 (2004).

${ }^{6}$ Y. Kondo, T. Suzuki, H. Inouye, K. Miura, T. Mitsuyu, and K. Hirao, Jpn. J. Appl. Phys., Part 2 37, L94-L96 (1998).

${ }^{7}$ A. Marcinkevičius, S. Juodkazis, M. Watanabe, M. Miwa, S. Matsuo, H. Misawa, and J. Nishii, Opt. Lett. 26, 277-279 (2001).

${ }^{8}$ C. Hnatovsky, R. S. Taylor, E. Simova, V. R. Bhardwaj, D. M. Rayner, and P. B. Corkum, Opt. Lett. 30, 1867-1869 (2005).

${ }^{9} \mathrm{H}$. Misawa and S. Juodkazis, Introduction in $3 D$ Laser Microfabrication: Principles and Applications (Wiley-VCH Verlag GmbH \& Co. KGaA, Weinheim, FRG, 2006).

${ }^{10}$ N. Bellini, K. Vishnubhatla, F. Bragheri, L. Ferrara, P. Minzioni, R. Ramponi, I. Christiani, and R. Osellame, Opt. Express 18, 4679-4688 (2010).

${ }^{11}$ D. Choudhury, W. T. Ramsay, R. Kiss, N. A. Willoughby, L. Paterson, and A. K. Kar, Lab Chip 12, 948-953 (2012).

${ }^{12}$ R. Osellame, H. J. W. M. Hoekstra, G. Cerullo, and M. Pollnau, Laser Photonics Rev. 5, 442-463 (2011).

${ }^{13}$ K. Sugioka, Y. Hanada, and K. Midorikawa, Laser Photonics Rev. 4, 386-400 (2010).

${ }^{14}$ Y. Bellouard and A. Said, Opt. Express 13, 6635-6644 (2005).

${ }^{15}$ S. Matsuo, Y. Tabuchi, T. Okada, S. Juodkazis, and H. Misawa, Appl. Phy. A 84, 99-102 (2006).

${ }^{16}$ S. Juodkazis, K. Nishimura, H. Misawa, T. Ebisui, R. Waki, S. Matsuo, and T. Okada, Adv. Mater. 18, 1361-1364 (2006).

${ }^{17}$ A. Rodenas, G. Zhou, D. Jaque, and M. Gu, Adv. Mater. 21, 3526-3530 (2009).

${ }^{18}$ A. Rodenas, A. Benayas, J. R. Macdonald, J. Zhang, D. Y. Tang, D. Jaque, and A. K. Kar, Opt. Lett. 36, 3395-3397 (2011).

${ }^{19}$ A. Rodenas, G. Zhou, D. Jaque, and M. Gu, Appl. Phys. Lett. 93, 151104 (2008).

${ }^{20}$ A. Ródenas, G. A. Torchia, G. Lifante, E. Cantelar, J. Lamela, F. Jaque, L. Roso, and D. Jaque, Appl. Phys. B 95, 85-96 (2009).

${ }^{21}$ W. A. Johnson, J. C. North, and R. Wolfe, J. Appl. Phys. 44, 4753-4757 (1973).

${ }^{22}$ P. J. T. Nunn, J. Olivares, L. Spadoni, P. D. Townsend, D. E. Hole, and B. J. Luff, Nucl. Instrum. Methods Phys. Res. B 127-128, 507-511 (1997). 